\title{
Skin and skin structure infections: treatment with newer generation fluoroquinolones
}

\author{
Philip Giordano' \\ Kurt Weber \\ Gail Gesin² \\ Jason Kubert' \\ 'Department of Emergency Medicine; \\ ${ }^{2}$ Department of Pharmacy, Orlando \\ Regional Medical Center, Orlando, \\ Florida, USA
}

\begin{abstract}
Skin and skin structure infections (SSSI) are an emerging issue in healthcare. They are responsible for increasing heathcare utilization, both in hospitalizations and intravenous antibiotic use. SSSI are caused by an evolving variety of pathogens, including Gram-positive, Gram-negative, and anaerobic bacteria. In combination with mounting resistance patterns, this diverse range of bacteria mandate empiric broad-spectrum antibiotic coverage. Historically, cephalosporins and penicillins have been the mainstay of treatment, but recent data suggest newer generation fluoroquinolones are being used with increasing frequency. In 2005, moxifloxacin joined gatifloxacin and levofloxacin as newer generation fluoroquionolones with Food and Drug Administration indications for SSSIs. Even within this group there exist subtle differences that impact optimal management. This paper offers the clinician a comparative review of the antimicrobial spectrum, pharmacodynamics, pharmacokinetics, and clinical efficacy data to support the appropriate use of fluoroquinolones in SSSIs.
\end{abstract}

Keywords: Uncomplicated skin and skin structure infections, complicated skin and skin structure infections, fluoroquinolone, moxifloxacin, gatifloxacin, levofloxacin

\section{Introduction}

Skin and skin structure infections (SSSI) represent a significant burden in infectious disease. They are responsible for approximately $10 \%$ of all hospital admissions (CDC 2001) and are the most common indication for intravenous antibiotics, accounting for 24 million days of therapy annually (AMR 2004). The approval of moxifloxacin for complicated skin and skin structure infections (cSSSI) by the Food and Drug Administration (FDA) in 2005 added another advanced generation fluoroquinolone to the array of antimicrobials with skin infection indications. This article addresses the evolving role of these newer generation fluoroquinolones in both uncomplicated (uSSSI) and complicated skin infections. Our goal is to provide an overview of the most commonly available newer generation fluoroquinolones that reside on hospital formularies along with the older generation ciprofloxacin. These are levofloxacin, moxifloxacin, and gatifloxacin (Table 1). Although Bristol-Myers Squibb Co., the manufacturer of gatifloxacin (Tequin $^{\circledR}$, Bristol-Myers Squibb Co., New York, NY), chose to suspend production of the drug in June of this year, as of the writing of this text, the ultimate fate of gatifloxacin remains undetermined. Furthermore, the authors believe that reviewing the properties, strengths, and limitations of gatifloxacin will aid both clinicians and research investigators in understanding the pharmacology of all fluoroquinolones. For this reason we have chosen to continue to include gatifloxacin in our comparison.

\section{Uncomplicated versus complicated infections}

The scope of SSSI is vast both in the range of anatomical structures affected and the pathogens involved. Bacterial skin infections can be divided into two categories, 
Table I Newer generation fluoroquinolones: basic facts

\begin{tabular}{llllll}
\hline Quinolone & Typical dose & Route of administration & Metabolism & Half life & Unique features \\
\hline Levofloxacin & $500-50 \mathrm{mg}$ & IV or PO & Renal & 6-9 hours & \\
Moxifloxacin & $400 \mathrm{mg}$ & IV or PO & Hepatic conjugation & I2 hours & Anaerobic coverage \\
Gatifloxacin & $400 \mathrm{mg}$ & IV or PO & Renal & $7-14$ hours & Dysglycemia \\
\hline
\end{tabular}

uncomplicated and complicated, as recognized in the FDA's Guidance for Industry (FDA 1998). Included in the uncomplicated category are simple abscesses, impetiginous lesions, furuncles, and cellulitis. Many traumatic wound infections can also be considered uncomplicated when they are the result of common skin colonizers that have entered through the break in the skin's natural defenses. These infections are typically monomicrobial and caused by Grampositive organisms (DiNubile and Lipsky 2004).

Complicated SSSIs characteristically involve deeper skin structures or coexist in patients with comorbidities or immune suppression (Nichols 1999). Included in this category are: major abscesses, infected burns and ulcers, infected bite wounds, and diabetic foot infections. Complicated infections may also include uncomplicated subtypes that are in anatomic locations that predispose them to polymicrobial infections, such as peri-anal abscesses. These infections are more likely to require surgical intervention and are more likely to be polymicrobial (DiNubile and Lipsky 2004). Other entities such as necrotizing fasciitis and infections involving prosthetic materials are generally not grouped with cSSSI because of their unique natures.

\section{Overview of microbiology of SSSI}

USSSIs are generally caused by Staphylococcus aureus and Streptococcus pyogenes, and empiric therapy is traditionally directed only toward these Gram-positive organisms (Guay 2003). First generation cephalosporins, commonly cephalexin, are the most frequently prescribed antibiotics. Extended spectrum cephalosporins, such as cefdinir, and dicloxacillin, an antistaphylococcal penicillin, are also appropriate choices (Stevens et al 2005). The newer generation fluoroquinolones levofloxacin, gatifloxacin, and moxifloxacin all have FDA-approved indications for uSSSI, but because of their broad spectrum potency are commonly reserved for more serious infections.

In recent years the emergence of community-acquired strains of methicillin resistant $S$. aureus (MRSA) has complicated the treatment of even uncomplicated infections. In some settings, community-acquired MRSA (CA-MRSA) represents greater than $50 \%$ of the $S$. aureus infections cultured from uSSSIs (Frazee et al 2005). These patients exhibit none of the commonly accepted risk factors for hospital-associated MRSA. Furthermore, these isolates represent a different genetic entity than the hospital-associated variety (Naimi et al 2003). An in-depth discussion of the emergence of CA-MRSA on treatment of uSSSIs is beyond the scope of this review, but clearly it has impacted the empiric choice of antibiotics in the primary care setting. CA-MRSA, while not sensitive to the usual choices for uncomplicated skin infections, displays in vitro sensitivity to a variety of other commonly prescribed antibiotics. While fluoroquinolones have shown variable efficacy both in vivo and in vitro against MRSA, the authors of this paper do not recommend the use of quinolones for MRSA. Several studies have, in fact, suggested that the use of quinolones may increase a patient's risk of developing a MRSA infection (Manhold 1998; Graffunder and Venezia 2002; Weber et al 2003). Trimethoprim/ sulfamethoxazole appears to be the most useful empiric treatment when CA-MRSA is suspected, demonstrating in vitro sensitivities of $100 \%$ in one study (Frazee et al 2005). Clindamycin can be used, but some MRSA carry a gene for inducible clindamycin resistance that begs caution with the use of this agent (Fiebelkorn et al 2003). Furthermore, as CA-MRSA is most common in abscesses and other pus containing infections, it is vitally important to determine those infections which can be managed by incision and drainage alone. Preliminary research in immunocompetent pediatric patients has suggested that uncomplicated abscesses less than $5 \mathrm{~cm}$ can be effectively managed with incision and drainage alone (Lee et al 2004). Evidence-based guidelines for determining which infections can be managed without antibiotic therapy are currently lacking for adults.

As in uSSSI, Gram-positive organisms are also the most common pathogens in cSSSI (Rennie et al 2003). However, Gram-negative and anaerobic microbes become more prevalent (Yee et al 2005). As illustrated in Figure 1, the most common Gram-negative organisms in cSSSIs include Pseudomonas aeruginosa, Escherichia coli, Klebsiella pneumoniae, and Enterobacter cloacae (Rennie et al 2003). Additionally, enteric flora, frequently enterococcus species and anerobes, become more important when the area of skin infected is in the lower half of the body (File and Tan 1995). The most common 


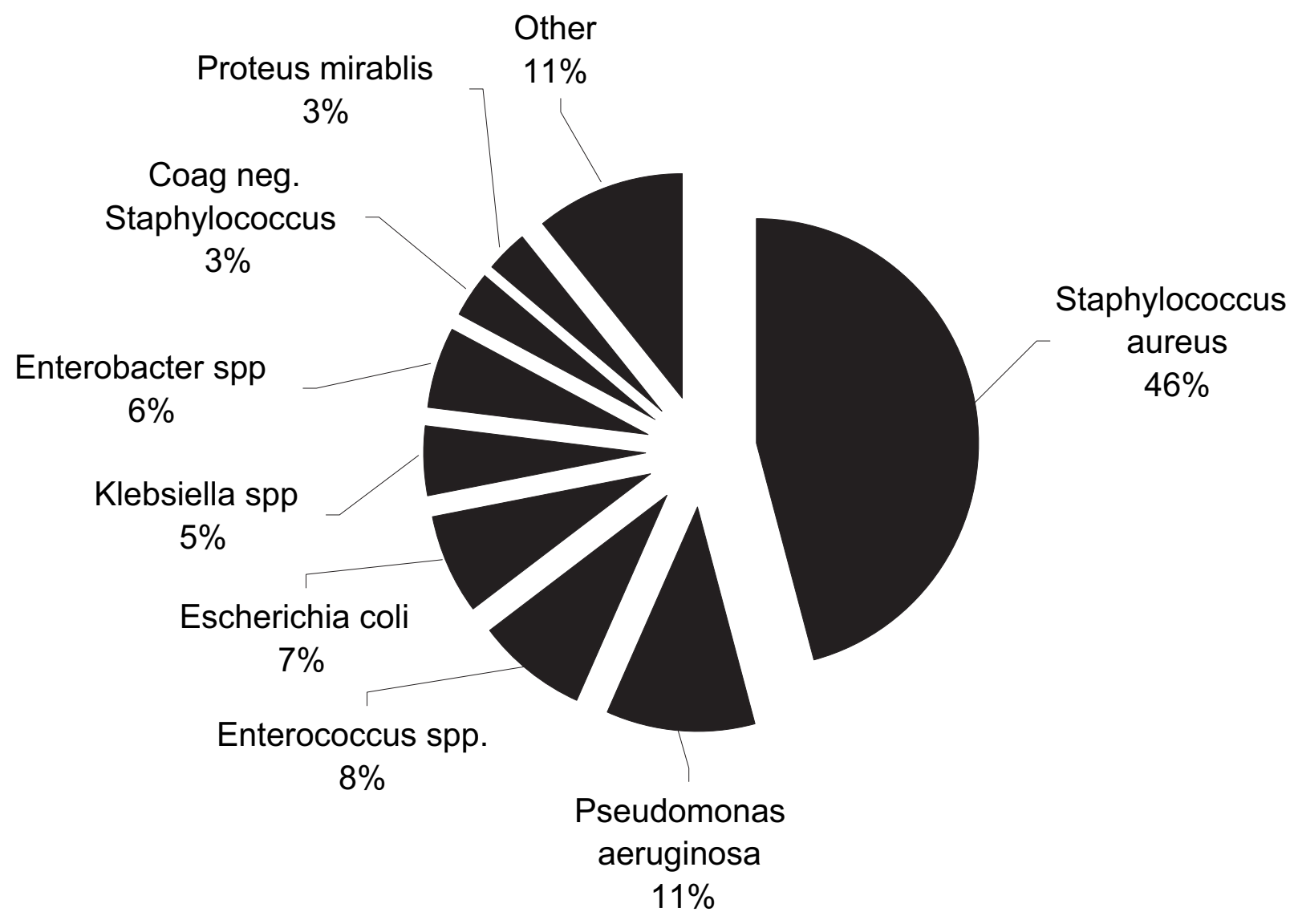

Figure I Prevalence of causative pathogens in complicated skin infections.

Reference: Rennie et al. 2003.

anaerobes isolated are typically Prevotella, Bacteroides, and Peptostreptococcus species. In one large study of complicated skin infections, these organisms were cultured in approximately $10 \%$ of infections (Giordano et al 2005).

Most of these patients with cSSSI are treated in the hospital with intravenous antimicrobial agents, but no consensus exists regarding empiric therapy (Rhagavan and Linden 2004). The most commonly used antimicrobials currently are broadspectrum cephalosporins and the combination penicillin/ penicillinase inhibitor agents (AMR 2004). The newer generation fluoroquinolones, with their broad-spectrum activity against Gram-positive and Gram-negative organisms, are important additions to battle against these infections, and can be used as monotherapy. Moxifloxacin in particular adds additional activity against anaerobic organisms that makes it a unique choice even amongst this group.

\section{Antimicrobial activity}

Moxifloxacin, gatifloxacin, and levofloxacin demonstrate excellent activity against the most common pathogens in skin infections, including $S$. aureus. The minimum concentration of drug needed to inhibit $90 \%$ of bacterial growth $\left(\mathrm{MIC}_{90}\right)$ for moxifloxacin and gatifloxacin against $S$. aureus is $0.125 \mathrm{mcg} / \mathrm{ml}$, which falls well below the Clinical and Laboratory Standards Institute (CLSI) breakpoint of $\leq 0.5$ $\mathrm{mcg} / \mathrm{mL}$ for susceptibility (Blondeau et al 2003; CLSI 2006). The $\mathrm{MIC}_{90}$ for levofloxacin against $S$. aureus is higher at 0.5 $\mathrm{mcg} / \mathrm{ml}$, but still falls below the CLSI susceptibility breakpoint of $\leq 1 \mathrm{mcg} / \mathrm{mL}$ (Blondeau et al 2003; CLSI 2006).

Although this data provides valuable information regarding in vitro antimicrobial activity, pharmacodynamic principles must also be considered. Since fluoroquinolones exhibit concentration-dependent killing, the area under the concentration curve to MIC (AUC-MIC) ratio is often used to correlate drug concentration and antimicrobial activity. In order to use this value clinically, interpretive breakpoints are needed. Unfortunately, these are not well established or validated clinically. While a target AUC-MIC breakpoint of $\geq 125$ is commonly stated for the fluoroquinolones, it is important to remember that this value was derived from a study in which ciprofloxacin was utilized for the treatment of primarily lower respiratory tract infections caused by a 
variety of organisms (Forrest et al 1993). Since it is likely that pharmacodynamic targets are both antibiotic- and pathogen-specific, a target of $\geq 125$ may not be applicable to all SSSI and their associated pathogens (Wright 2000).

In an attempt to overcome this problem, a comparison was undertaken by Firsov and colleagues (2005). Using two clinical isolates of $S$. aureus, fluoroquinolone-specific and strain-independent AUC-MIC relationships were evaluated. Additionally, AUC-MIC breakpoints for each agent were predicted based on antimicrobial effect. The breakpoints for moxifloxacin, gatifloxacin, and levofloxacin were 75,95 , and 115 respectively. AUC- $\mathrm{MIC}_{50}$ ratios were calculated following standard dosing and $\mathrm{MIC}_{50} \mathrm{~s}$ from the strains of $S$. aureus. These values were 206, 143, 65, and 118 for moxifloxacin, gatifloxacin, levofloxacin (500 mg) and levofloxacin (750 mg), respectively. As can be seen in Table 2, standard dosing was sufficient to exceed the established breakpoints for all agents except levofloxacin at the $500 \mathrm{mg}$ dose. Incorporating the above pharmacodynamic findings with published MIC data, one can see that the results are similar. While Firsov and colleague's experiment is stylish and well thought out, correlation with clinical efficacy is necessary.

Gatifloxacin and levofloxacin demonstrate excellent in vitro activity against S.s pyogenes with $\mathrm{MIC}_{90} \mathrm{~s}$ well below the CLSI breakpoints for susceptibility of $\leq 1 \mathrm{mcg} / \mathrm{mL}$ and $\leq 2 \mathrm{mcg} / \mathrm{mL}$, respectively (Milatovic et al 2000; Blondeau et al 2003; CLSI 2006). Moxifloxacin demonstrates good activity against this organism as well (Milatovic et al 2000). In vitro activity also appears to be excellent for gatifloxacin and moxifloxacin against Enterococcus faecalis based on $\mathrm{MIC}_{90}$ data, although interpretive standards are not available (Edmiston et al 2004; CLSI 2006). Perhaps as expected, none of the newer generation quinolones show reliable in vitro activity against the hardy Enterococcus faecium, with $\mathrm{MIC}_{90} \mathrm{~s}$ of 8 or higher.

All three agents have good in vitro activity against the three most common nonpseudomonal Gram-negatives found in complicated skin infections (Blondeau et al 2003; Edmiston et al 2004; CLSI 2006). These include Escherichia coli, Klebsiella pneumoniae and Enterobacter cloacae. In comparison with moxifloxacin, gatifloxacin and levofloxacin exhibit greater activity against Pseudomonas species (Blondeau et al 2003) and therefore moxifloxacin should not be used as monotherapy against this organism.

Moxifloxacin is distinguished from gatifloxacin and levofloxacin by its added activity against anaerobes. In vitro pharmacokinetic modeling provides some insight as to the target AUC-MIC ratios for this agent against several anaerobic
Table 2 AUC/MIC90 ratios for various flouroquinolones against Staphylococcus aureus

\begin{tabular}{llll}
\hline Quinolone & $\begin{array}{l}\text { Dose } \\
(\mathbf{m g})\end{array}$ & $\begin{array}{l}\text { AUC } \\
(\mathbf{m c g} \cdot \mathbf{h r} / \mathbf{m L})\end{array}$ & $\begin{array}{l}\text { MIC } \\
(\mathbf{m c g} / \mathbf{m L})\end{array}$ \\
\hline Gatifloxacin & 400 & 32.9 & 0.125 \\
Levofloxacin & 500 & 45.6 & 0.5 \\
Levofloxacin & 750 & 82.6 & 0.5 \\
\hline
\end{tabular}

Adapted from Firsov AAC 2005;49:2642 (AUC data) and Blondeau Int J Antimicrobial Agents 2003;22:147 ( MIC $_{90}$ data)

species. A 90\% effect was achieved with an AUC-MIC ratio of 25.7 for Bacteroides fragilis, 16.2 for Clostridium perfringens, and 17.4 for Gram-positive anaerobic cocci (Noel 2005). Integrating this data with moxifloxacin's reported AUC of 48 following a single $400 \mathrm{mg}$ dose, it is evident in vitro data supports moxifloxacin's clinical success against anaerobic organisms in several recent trials (Edmiston et al 2004; Giordano et al 2005).

\section{Pharmacokinetic profile}

The pharmacokinetics of moxifloxacin, gatifloxacin, and levofloxacin are similar (Table 2). All are well absorbed following oral administration with a bioavailability of approximately $90 \%$ (Wickersham and Schweain 2005). Oral and intravenous doses for these agents are the same. Although food does not significantly affect bioavailability, aluminumand magnesium-containing antacids, other metal cations such as iron and zinc, as well as various buffered didanosine preparations and sucralfate can decrease oral absorption (Wickersham and Schweain 2005). The most stringent interval restrictions apply to moxifloxacin, which should be administered at least four hours before or eight hours after the above-mentioned agents. Gatifloxacin should be given at least four hours prior to these interacting medications and levofloxacin should be administered at least two hours before or after (Lubasch et al 2000; Furlanut et al 2003; Wickersham and Schweain 2005).

Moxifloxacin, gatifloxacin, and levofloxacin follow linear pharmacokinetics, meaning that serum concentrations change in proportion to dose. The approximate half-lives for moxifloxacin (12 hours), gatifloxacin (7 to 14 hours), and levofloxacin (6 to 9 hours) are all sufficiently long to allow for the convenience of once daily dosing. All reach steadystate serum concentrations within three days and are widely distributed throughout the body, with tissue concentrations often exceeding those in the plasma (Wickersham and Schweain 2005). In a pharmacokinetic study evaluating the 
penetration of moxifloxacin into inflammatory fluid, a 400 $\mathrm{mg}$ dose achieved mean blister fluid penetration rates of $83.5 \%$ and $93.7 \%$ following oral and intravenous administration, respectively (Wise et al 1999). An examination of the maximum AUC values for blister fluid and plasma revealed that penetration into blister fluid exceeded $100 \%$. Gatifloxacin and levofloxacin also exhibit good penetration into blister fluid (Trampuz et al 2000; Trampuz and Laifer 2002).

The most important pharmacokinetic differences between these agents are their metabolic and elimination pathways. Specifically, moxifloxacin is primarily eliminated via hepatic conjugation while gatifloxacin and levofloxacin are eliminated renally. An assessment of end-organ function is therefore necessary for proper dosing and avoidance of toxicity. Moxifloxacin is metabolized by hepatic sulfate and glucuronide conjugation, accounting for $38 \%$ and $14 \%$ of a dose, respectively (Wickersham and Schweain 2005). A beneficial property of this agent is that it is not metabolized by the cytochrome $\mathrm{p}-450$ enzyme system, thereby minimizing the potential for drug interactions. As $95 \%$ of an oral dose is either excreted as unchanged drug or known metabolites, no dosage adjustment is required in patients with impaired renal function (Wickersham and Schweain 2005). Additionally, no dosage adjustment in needed in patients with mild to moderate hepatic insufficiency, defined as Child-Pugh class A or B. However, moxifloxacin is not recommended in patients with severe hepatic insufficiency.

Gatifloxacin and levofloxacin are primarily removed renally as unchanged drug via glomerular filtration and tubular secretion. In patients with a creatinine clearance less than $40 \mathrm{~mL} / \mathrm{min}$, gatifloxacin doses should be reduced from $400 \mathrm{mg}$ daily to $400 \mathrm{mg}$ initially followed by $200 \mathrm{mg}$ daily (Wickersham and Schweain 2005). This dose reduction also applies for those on hemodialysis or continuous ambulatory peritoneal dialysis (CAPD). Doses should always be administered just after hemodialysis. Levofloxacin must also be adjusted for renal impairment. It is important to note that dose adjustment for levofloxacin is dependent on indication as well as creatinine clearance (Wickersham and Schweain 2005). For uncomplicated SSSI in patients with a creatinine clearance of 20 to $49 \mathrm{~mL} / \mathrm{min}$, a $500 \mathrm{mg}$ dose should be followed by $250 \mathrm{mg}$ every 24 hours. For those with a creatinine clearance less than $20 \mathrm{~mL} / \mathrm{min}$ the same initial dose should be followed by $250 \mathrm{mg}$ every 48 hours. This extended interval also applies to patients on hemodialysis or CAPD. For cSSSI in patients with a creatinine clearance of 20 to $49 \mathrm{~mL} / \mathrm{min}$, a $750 \mathrm{mg}$ initial dose should be followed by $750 \mathrm{mg}$ every 48 hours. For those with a creatinine clearance less than $20 \mathrm{~mL} /$ min, including patients on hemodialysis or CAPD, a $750 \mathrm{mg}$ initial dose should be followed by $500 \mathrm{mg}$ every 48 hours.

\section{Clinical studies}

Table 3 summarizes the available clinical trials of newer generation fluoroquinolones for skin and skin structure infections.

\section{Moxifloxacin}

Parish and colleagues (2005) studied a seven-day course of oral moxifloxacin (400 mg, once daily) versus cephalexin (500 mg, three times daily) in a prospective, randomized, blinded trial for uSSSI. The most common infections included cellulitis, impetigo, and minor wound infections. 351 patients were included in the clinically evaluable group. The clinical response rate (resolution of symptoms) was $90 \%$ in the moxifloxacin group versus $91 \%$ in the cephalexin group. 125 patients had bacteriologic data available; 68 in the moxifloxacin group and 57 for cephalexin. By far the most prevalent organism isolated was S. aureus, followed by Streptococcus spp. Eradication rates in the S. aureus isolates were $92 \%$ and $93 \%$, for moxifloxacin and cephalexin, respectively.

Giordano et al (2005) compared intravenous/oral moxifloxacin (400 $\mathrm{mg}$ once daily) with iv piperacillintazobactam $(3.0 / 3.75 \mathrm{~g}$ every 6 hours $)$ followed by oral amoxicillin-clavulanate ( $800 \mathrm{mg}$ twice daily) in cSSSI.

Table 3 Summary of clinical trials of newer generation fluoroquinolones for skin and skin structure infections

\begin{tabular}{|c|c|c|c|c|c|}
\hline Author and year & Type of skin infection & $\begin{array}{l}\text { Study drug and } \\
\text { dosing }\end{array}$ & $\begin{array}{l}\text { Duration of } \\
\text { therapy }\end{array}$ & $\begin{array}{l}\text { Clinical } \\
\text { cure rate }\end{array}$ & $\begin{array}{l}\text { Bacteriological } \\
\text { cure rate }\end{array}$ \\
\hline Parrish 2005 & Uncomplicated & Moxifloxacin 400 mg PO daily & 7 days & $90 \%$ & $92 \%$ \\
\hline Giordano et al 2005 & Complicated & Moxifloxacin $400 \mathrm{mg}$ IV/PO daily & $7-14$ days & $79 \%$ & $78 \%$ \\
\hline Tarshis et al 200I & Uncomplicated & Gatifloxacin 400 mg PO daily & $7-10$ days & $91 \%$ & $91 \%$ \\
\hline Tarshis et al 200I & Uncomplicated & Levofloxacin 500 mg PO daily & $7-10$ days & $84 \%$ & $84 \%$ \\
\hline Nichols et al 1997 & Uncomplicated & Levofloxacin 500 mg PO daily & $7-10$ days & $98 \%$ & $98 \%$ \\
\hline Graham et al 2002 & Complicated & Levofloxacin 750 mg IV/PO daily & 7-14 days & $84 \%$ & $91 \%$ \\
\hline
\end{tabular}


Treatment duration was between 7 and 14 days, at the investigator's discretion. Infection types enrolled were complicated by definition, and included deep abscesses, cellulitis, diabetic foot infections, infected ischemic/decubitus ulcers, and surgical wound infections. The clinical cure rates, $79 \%$ (moxifloxacin) and $82 \%$ (control group), in the efficacy valid population $(n=367)$ were not statistically different. The microbiologically valid population $(\mathrm{n}=237)$ grew a range of pathogens. The most common was S. aureus (52\%), followed by nongroup A streptococci, Enterococcus, Strep pyogenes, Peptostreptococci spp., and Escherichia coli. MRSA was found in 7\% of infections. Polymicrobial infections were found in 113 patients (47\%). Bacterial eradication rates were similar in both groups for all targeted pathogens, including $81 \%$ for MSSA. Eradication rates in monomicrobial infections ( $85 \%$ both groups) were higher than polymicrobial infections ( $70 \%$ moxifloxacin vs $77 \%$ control).

\section{Gatifloxacin}

In a double-blind, randomized study, Tarshis and colleagues (2001) compared gatifloxacin (400 mg once daily) versus levofloxacin (500 $\mathrm{mg}$ once daily) in uSSSI. Total duration of therapy was $7-10$ days. In the clinically evaluable population $(\mathrm{n}=333)$, the cure rate for gatifloxacin and levofloxacin was $91 \%$ and $84 \%$, respectively. The types of infection included were similar to other uSSSI studies and included near equal distribution between folliculitis, wound infections, cellulitis, and abscesses. Across these diagnoses, higher clinical cure rates trended toward gatifloxacin but were not statistically significant. Of the 308 pathogens isolated, the most common were $S$. aureus, S. pyogenes, and S. agalactiae. Microbiological eradication rates mirrored clinical cure rates and were not statistically different for any pathogen.

\section{Levofloxacin}

In addition to the Tarshis study, data for levofloxacin in uSSSI comes from trials against ciprofloxacin. Nichols and colleagues (1997) compared 7-10 days course of levofloxacin (500 mg once daily) against ciprofloxacin (500 mg twice daily) in an open label, randomized, multi-center trial. Infection types enrolled were largely consistent with prior uSSSI trials, noting a predominance of cellulitis (43\%). In the clinically assessable population $(n=375)$, the clinical success rate (cured plus improved) was $98 \%$ and $94 \%$ for levofloxacin and ciprofloxacin respectively. This difference was not statistically significant. A total of 519 pathogens were isolated, with $S$. aureus and $S$. pyogenes being the most prevalent. The difference in overall bacterial eradication rates between levofloxacin (98\%) and ciprofloxacin (89\%) was not statistically significant.

Evidence for levofloxacin in cSSSI comes from 1 large multicenter, open label, randomized clinical trial. Graham and colleauges (2002) compared levofloxacin ( $750 \mathrm{mg}$, intravenous/ oral) against ticarcillin-clavulanate (TC) (3.1 g intravenous every 4-6 hours) alone or followed by amoxicillin-clavulanate (AC) (875 mg every 12 hours). Duration of therapy was 7-14 days as clinically indicated. Both regimens were equally efficacious. The clinical success rates, $84 \%$ (levofloxacin) and $80 \%$ (TC/AC), were not statistically different. A diverse range of pathogens were cultured, including Gram-positives (Staph, Strep, Enterococcus), Gram-negatives (Proteus, Enterobacter, E. coli, Pseudomonas), and anaerobes. Overall microbiologic eradication rates, $91 \%$ (levofloxacin) and 84\% (TC/AC), were similar.

\section{Safety and tolerability}

Moxifloxacin, at the $400 \mathrm{mg}$ dose, has been widely shown to be safe and well tolerated in numerous clinical trials across many disease types. In pooled data from over 8600 patients who received the $400 \mathrm{mg}$ dose, most adverse events were described as mild to moderate in severity and required no treatment. The most common adverse events considered by investigators to be at least possibly drug-related included nausea $(6 \%)$, diarrhea $(5 \%)$, dizziness $(2 \%)$. Moxifloxacin was discontinued due to drug related adverse reactions in $2.9 \%$ and $6.3 \%$ of patients for oral and intravenous administration respectively (Bayer Pharmaceuticals 2005). Similar findings have emerged from clinical trials involving gatifloxacin. In trials that included over 5000 patients, the majority of adverse effects were considered to be mild, and therapy was discontinued due to adverse events in only $2.7 \%$ of patients. Nausea (8\%), vaginitis $(6 \%)$, diarrhea $(4 \%)$, headache (3\%), and dizziness (3\%) occurred most commonly (Bristol-Myers Squibb 2006a). In phase III clinical trials evaluating levofloxacin, approximately $4 \%$ of patients discontinued therapy due to adverse events. These events included nausea (6.8\%), headache (5.8\%), diarrhea (5.4\%), insomnia (4.6\%), and constipation (3.1\%); all comparable with placebo (Ortho-McNeil Pharmaceuticals 2005).

Although the agents discussed in this paper are generally well tolerated, the impact of fluoroquinolones on the QT interval continues to be debated in the literature. The effects of QT prolongation can range from incidental electrocardiogram findings to life-threatening arrhythmias, specifically, torsades de pointes (TdP). Fluoroquinolones are thought to prolong 
the QT interval through interaction with outward potassium channels in myocardial tissue. Preclinical research shows the effect of various quinolones on the QT interval appears to vary quite markedly for each agent (Hagiwara et al 2001). The most significant changes were seen with sparfloxacin, which has since been discontinued partly as a result of these findings. Moxifloxacin, gatifloxacin, and levofloxacin did not show any significant effects on the duration of the action potential at typical therapeutic concentrations (Hagiwara et al 2001; Yap and Camm 2003). While these models provide a baseline understanding of the quinolones' impact on the QTc, it is important to discern what the clinical relevance of this data is considering the multifactorial nature of cardiac arrhythmias. Based on the European Union's Committee for Proprietary Medicinal Products guidelines, a new drug that induces QTc changes of less than $30 \mathrm{msec}$ from baseline is unlikely to raise significant concern regarding the potential development of arrhythmias (EMEA 2005). A risk stratification schedule for torsades de pointes with respect to antimicrobial agents has been developed (Owens 2004). This stratification integrates nonclinical models, clinical studies, case reports, pharmacokinetic data, and post-marketing safety studies with portions of a previously developed risk assessment scheme. According to this pool of data, medications are divided into five schedules, with schedule I associated with the highest risk of TdP and schedule $\mathrm{V}$ the lowest. Moxifloxacin, gatifloxacin, and levofloxacin are classified as schedule IV agents, meaning that the risk for TdP is minimal, and the drugs display a favorable pharmacokinetic safety profile. Such features include predictable pharmacokinetics, more than one elimination pathway, and/or weak interference with the cytochrome p-450 enzyme system.

Multiple case reports that have highlighted the development of serious ventricular arrhythmias associated with the administration of the new quinolones have drawn attention to important predisposing risk factors for QTc prolongation (Iannini and Circiumaru 2001; Ball 2002; Bertino et al 2002; Gandhi et al 2003; Amankwa et al 2004). These reports illustrate that clinically significant arrhythmias tend to develop in patients with risk factors such as known syndromes of prolonged QTc, severe hypokalemia or hypomagnesemia, and concurrent use of other drugs that are known to prolong the QTc interval, such as Class IA or III antiarrhythmics. Consensus has been reached that quinolones are best avoided in such high-risk patients (Owens 2001).

In addition to cardiac effects, gatifloxacin has recently received attention for its potential to induce both hypo- and hyperglycemia. As a result, the prescribing information for gatifloxacin was revised to include a contraindication in patients with diabetes mellitus (Bristol-Myers Squibb 2006b). This decision was based on post-marketing case reports of significant hypoglycemia and hyperglycemia, primarily in diabetic patients (Bristol-Myers Squibb 2006b). However, dysglycemia has also been reported in patients without a history of diabetes, emphasizing the importance of appropriate patient selection and monitoring when prescribing gatifloxacin. As mentioned in the introduction, Bristol-Myers Squibb Co. has now chosen to suspend production of gatifloxacin. It is important to note the US FDA did not mandate the discontinuation of gatifloxacin, nor did the FDA require a "black box"warning for dysglycemia. In a statement issued by BristolMyers Squibb Co., the drug manufacturer states that "the cost to manufacture outweighed the usage rate" and continued production of gatifloxacin was no longer cost-effective.

An increasingly common consequence of antimicrobial use is the development of Clostridium difficile associated disease. C. difficile infections can range in severity from mild diarrheal illness to life-threatening toxic megacolon and intraabdominal sepsis. Many studies have investigated the complex and overlapping set of risk factors that may predispose a patient to develop $C$. difficile infections, and while much controversy remains, a few overriding themes have emerged. The first risk is the use of any antimicrobial agent, followed by environmental exposure to the bacteria, with the extent of infection dependent upon a host other factors including the use of gastric acid reducing medications, underlying comorbidities, advanced age, and host serum immunoglobulin levels (Mulligan et al 1993; Bignardi 1998; Johnson and Gerding 1998; Dial et al 2004; Gerding et al 2004). Predictably, the fluoroquinolones, as a commonly prescribed class of antimicrobials, have been implicated as a risk factor for $C$. difficile infections, particularly during local outbreaks of resistant strains (Loo et al 2005). However, since nearly all antimicrobial agents have been associated with the development of $C$. difficile infections, one must ask if the fluoroquinolones as a class result in more infections than other classes of antimicrobials. A case-control study presented at the American Society of Microbiology in 2005 found a relative risk of developing $C$. difficile of 3.2 for fluoroquinolones, compared with relative risks of $14,5.5$, and 4.2 for cephalosporins, macrolides, and vancomycin respectively (Owens 2006). Other speculation has suggested that antimicrobials with activity against anaerobic organisms would differentially favor the growth of $C$. difficile. This, 
however, has not been borne out in numerous studies. Clinical studies of the anti-anaerobic moxifloxacin which involved more than 21000 patients did not show any increased incidence of $C$. difficile infections in patients treated with moxifloxacin relative to patients treated with other antimicrobials (Ball et al 2004; Owens 2006). Furthermore, a comparison of moxifloxacin against levofloxacin, which does not possess activity against anaerobes, failed to show any difference in rates of $C$. difficile infections (Anzueto et al 2006). While all clinicians should be wary of precipitating C. difficile-associated disease when prescribing antimicrobials, at present there exists no evidence to suggest that any class is immune from the risk (Owens 2006).

\section{Conclusion}

With the addition of moxifloxacin to the ranks of antimicrobials with an indication for skin and skin structure infections, most clinicians have a newer generation fluoroquinolone available to them on their hospital formulary. This is, of course, in addition to the litany of drugs with antimicrobial spectra that overlap the list of common skin pathogens. With their once daily convenience, easy transition to oral therapy and excellent safety profile, the newer generation fluoroquinolones are excellent additions to the current armamentarium against SSSIs. However, while moxifloxacin, gatifloxacin, and levofloxacin have proven themselves to be effective agents across the full gamut of skin and skin structure infections, clinicians should be prudent in the use of flouroquinolones as first line agents. Their efficacy against a broad variety of less common Gramnegatives for which current antimicrobial choices are limited and dwindling needs to be preserved. The utility of these agents lies in their ability to serve as monotherapy in the face of polymicrobial infections where Gram-negative organisms are suspected along with the usual gram positive culprits. Moxifloxacin provides an additional benefit in its coverage of anaerobes, and its role in the treatment of these infections is likely to expand.

\section{Reference}

Amankwa K, Krishnan SC, Tisdale JE. 2004. Torsades de pointes associated with fluoroquinolones: importance of concomitant risk factors. Clin Pharmacol Ther, 75:242-7.

Anzueto A, Niederman MS, Pearle J, et al. 2006. Community acquired pneumonia recovery in the elderly (CAPRIE): efficacy and safety of moxifloxacin therapy versus that of levofloxacin therapy. Clin Infect Dis, 42:73-81.

[AMR] Arlington Medical Resources. 2004. Hospital Antibiotic Market Database [online]. Accessed on 25 July 2006. URL: http://www.amr-data.com/.
Ball P, Stahlman R, Kubin R, et al. 2004. Safety profile of oral and intravenous moxifloxacin: cumulative data from clinical trials and postmarketing studies. Clin Ther, 26:940-50.

Ball P. 2000. Quinolone-induced QT interval prolongation: a not-sounexpected class effect. J Antimicrob Chemother, 45:557-9.

Bayer Pharmaceuticals Co. 2005. Avelox (moxifloxacin) package insert. Westhaven, CT: Bayer Pharmaceuticals.

Bignardi GE. 1998. Risk factors for Claustridium difficile infection. J Hosp Infect, 40:1-15.

Bertino JS, Owens RC Jr., Carnes TD, et al. 2002. Gatifloxacin-associated corrected QT interval prolongation, torsades de pointes, and ventricular fibrillation in patients with known risk factors. Clin Infect Dis, 34:861-3.

Blondeau JM, Hansen G, Metzler KL, et al. 2003. In vitro susceptibility of 4903 bacterial isolates to gemifloxacin and advanced fluoroquinolones. Int J Antimcrob Agents, 22:147-54.

Bristol-Myers Squibb Co. 2006a. Tequin (gatifloxacin) package insert. Princeton, NJ: Bristol-Myers Squibb Co.

Bristol-Myers Squibb Co. 2006b. Dear Health Care Provider letter [online]. Accessed 10 April 2006. URL: www.fda.gov/medWatch/safety/2006/ tequin_DHCP.pdf.

[CDC] Center for Disease Control. 2001. Incidence of soft tissue infections: San Francisco General Hospital-1996-2000. MMWR, 50:381-4.

[CLSI] Clinical and Laboratory Standards Institute. 2006. Performance standards for antimicrobial susceptibility testing. Sixteenth Informational Supplement. CLSI document M100-S16.

Dial S, Alrasadi K, Huang A, et al. 2004. Risk of Clostridium dificile diarrhea among hospital inpatients prescribed proton pump inhibitors: cohort and case-control studies. Can Med Assoc J, 171:33-8.

DiNubile MJ, Lipsky BA. 2004. Complicated Infections of skin and skin structure infections: When infection is more than skin deep. JAntimicrob Chemother, 53:37-50.

Edmiston CE, Krepel CJ, Seabrook GR, et al. 2004. In vitro activities of moxifloxacin against 900 aerobic and anaerobic surgical isolates from patients with intra-abdominal and diabetic foot infections. Antimicrob Agents Chemother, 48:1012-16.

[EMEA] European Agency for the Evaluation of Medicinal Products; Committee for Proprietary Medicinal Products. 2006. Points to consider: the assessment of the potential for QT interval prolongation by noncardiovascular medicinal products [online]. Accessed 10 April 2006. URL: www.coresearch.biz/regulations/cpmp.pdf.

[FDA] Food and Drug Administration. 1998. Guidance for industry: uncomplicated skin and skin structure infections--Developing antimicrobial drugs for therapy [online]. Accessed 10 April 2005. URL: www.fda.gov/cder/guidance/2566dft.pdf.

Fiebelkorn KR, Crawford SA, McElmeel ML, et al. 2003. Practical disc diffusion method for detection of inducible clindamycin resistance in Staphylococcus aureus and coagulase-negative staphylococci. J Clin Microbiol, 41:4740-4.

File TM Jr. Tan JS. 1995. Treatment of skin and soft-tissue infections. Am J Surgery, 169(5A Suppl):27S-33S.

Firsov AA, Lubenko IY, Vostrov SN, et al. 2005. Antistaphylococcal effect related to the area under the curve/MIC ratio in an in vitro dynamic model: predicted breakpoints versus clinically achievable values for seven fluoroquinolones. Antimicrob Agents Chemother, 49:2642-7.

Forrest A, Nix DE, Ballow CH, et al. 1993. Pharmocodynamics of intravenous ciprofloxacin in seriously ill patients. Antimicrob Agents Chemother, 37:1073-81.

Frazee BW, Lynn J, Charlebois ED, et al. 2005. High prevalence of methicillin-resistant Staphylococcus aureus in emergency department skin and soft tissue infections. Ann Emerg Med, 45:311-20.

Furlanut M, Brollo L, Lugatti,E, et al. 2003. Pharmacokinetic aspects of levofloxacin $500 \mathrm{mg}$ once daily during sequential intravenous/ oral therapy in patients with lower respiratory tract infections. $J$ Antimicrob Chemother, 51:101-6. 
Gandhi PJ, Menezes PA, Vu HT, et al. 2003. Fluconazole- and levofloxacin-induced torsades de pointes in an intensive care unit patient. Am J Health-Syst Pharm, 60:2479-83.

Gerding DN. 2004. Clindamycin, cephalosporins, fluoroquinolones, and Claustridium difficile-associated: this is an antimicrobial resistance problem. Clin Infect Dis, 38:646-8.

Giordano P, Song J, Pertel P, et al. 2005. Sequential intravenous/oral moxifloxacin versus intravenous pipericillin-tazobactam followed by oral amoxicillin-clavulanate for the treatment of complicated skin and skin structure infection. Inter J Antimicro Agents, 26:35765 .

Graffunder EM, Venezia RA. 2002. Risk factors associated with nosocomial methicillin-resistant Staphylococcus aureus (MRSA) infection including previous use of antimicrobials. JAntimicrob Chemother, 49:999-1005.

Graham DR, Talan DA, Nichols RL, et al. 2002. Once-daily, high dose levofloxacin versus ticaricillin-clavulanate alone or followed by amoxicillin for complicated skin and skin structure infections: A randomized, open label trial. Clin Inf Dis, 35:381-9.

Guay D. 2003. Treatment of bacterial skin and skin structure infections. Expert Opin Pharmacother, 4:1259-75.

Hagiwara T, Satoh S, Kasai Y, et al. 2001. A comparative study of the various fluoroquinolone antibacterial agents on the cardiac action potential in guinea pig right ventricular myocardium. Jpn J Pharmacol, 87:231-4.

Iannini PB, Circiumaru I. 2001. Gatifloxacin-induced QTc prolongation and ventricular tachycardia. Pharmacotherapy, 21:361.

Johnson S, Gerding DN. 1998. Clostridium difficile-associated diarrhea. Clin Infect Dis, 26:1027-34.

Lee MC, Rios AM, Aten MF, et al. 2004. Management and outcomes of skin and soft tissue abcesses coused by community acquired methicillin resistant Staphylococcus aureus. Pediatri Infect Dis J, 23:123-7.

Loo VG, Poirier L, Miller MA, et al. 2005. A predominantly clonal multiinstitutional outbreak of Clostridium difficile-associated diarrhea with high morbidity and mortality. N Engl J Med, 353(23):2442-9.

Lubasch A, Keller I, Borner K, et al. 2000. Comparative pharmacokinetics of ciprofloxacin, gatifloxacin, grepafloxacin, levofloxacin, trovafloxacin, and moxifloxacin after single oral administration in healthy volunteers. Antimicrob Agents and Chemother, 44:2600-3.

Manhold C, von Rolbieki U, Brase R, et al. 1998. Outbreaks of Staphylococcus aureus infections during treatment of late onset pneumonia with ciprofloxacin in a prospective randomized study. Intensive Care Med, 24:1327-30.

Milatovic D, Schmitz F, Brisse S, et al. 2000. In vitro activities of sitafloxacin (DU-6859a) and six other fluoroquinolones against 8,796 clinical bacterial isolates. Antimicrob Agents Chemother, 44:1102-7.

Mulligan ME, Miller SD, McFarland, et al. 1993. Elevated levels of serum immunoglobulins in asymptomatic carriers of Clostridium difficile. Clin Infect Dis. 16(suppl 4):S239-44.

Naimi TS, LeDell KH, Como-Sabetti K, et al. 2003. Comparison of community- and health care-acquired methicillin resistant Staphylococcus aureus infection. JAMA, 290:2976-84.

Nichols RL, Smith JW, Gentry LO, et al. 1997. Multi-center, randomized study comparing levofloxacin and ciprofloxacin for uncomplicated skin and skin structure infections. South Med J, 90:1193-200.

Nichols RL. 1999. Optimal treatment of complicated skin and skin structure infections. J Antimicrob Chemother, 44:19-23.
Noel AR, Bowker KE, Macgowan AP. 2005. Pharmacodynamics of moxifloxacin against anaerobes studied in an in vitro pharmacokinetic model. Antimicrob Agents Chemother, 49:4234-9.

Ortho-McNeil Pharmaceutical. 2005. Levaquin (levofloxacin) package insert. Raritan, NJ

Owens RC. 2001. Risk assessment for antimicrobial agent-induced QTc interval prolongation and torsades de pointes. Pharmacotherapy, 21:301-19.

Owens RC. 2004. QT prolongation with antimicrobial agents: understanding the significance. Drugs, 64:1091-124.

Owens RC. 2006. Claustridium difficile- associated disease: An emerging threat to patient safety. Pharmacotherapy, 26(3): 299-311.

Parish LC, Routh HB, Miskin B, et al. 2000. Moxifloxacin versus cephalexin in the treatment of uncomplicated skin infections. Int J Clin Pract, $54: 497-503$

Raghavan R, Linden PK. 2004. Newer treatment options for skin and soft tissue infections. Drugs, 64:1621-42.

Rennie RP, Jones RN, Mutnick AH, et al. 2003. Occurrence and antimicrobial susceptibility patterns of pathogens isolated from skin and soft tissue infections: report from the Sentry antimicrobial surveillance program. Diagn Microbio Infect Dis, 45:287-93.

Stevens DL, Bisno AL, Chambers HF. 2005. Practice guidelines for the diagnosis and management of skin and soft-tissue infections. Clin Infect Dis, 41:1373-406.

Tarshis GA, Miskin BM, Jones TM, et al. 2001. Once-daily oral gatifloxacin versus oral levofloxacin in treatment of uncomplicated skin and soft tissue infections: double-blind, multicenter, randomized study. Antimicrob Agents Chemother, 45:2358-62.

Trampuz A, Laifer G. 2002. Pharmacokinetics and pharmacodynamics of gatifloxacin against Streptococcus pneumoniae and Staphylococcus aureus in human skin blister fluid. Antimicrob Agents Chemother, 46:3630-3.

Trampuz A, Wenk M, Rajacic Z, et al. 2000. Pharmacokinetics and pharmacodynamics of levofloxacin against Streptococcus pneumoniae and Staphylococcus aureus in human skin blister fluid. Antimicrob Agents Chemother, 44:1352-5.

Weber SG, Gold HS, Hooper DC, et al. 2003. Fluoroquinolones and the risk of methicillin resistant Staph aureus in hospitalized patients. Emerg Infect Dis, 9:1415-22.

Wickersham RM, Schweain SL (eds). 2005. Drug facts and comparisons. St. Louis: Wolters Kluwer Health, Inc.

Wise R, Andrews JM, Marshall G, et al. 1999. Pharmacokinetics and inflammatory-fluid penetration of moxifloxacin following oral and intravenous administration. Antimicrob Agents and Chemother, 43:1508-10.

Wright DH, Brown GH, Peterson ML, et al. 2000. Application of fluoroquinolone pharmacodynamics. J Antimocrob Chemother, 46:669-83.

Yap YG, Camm AJ. 2003. Drug-induced QT prolongation and torsades de pointes. Heart, 89:1363-72.

Yee SY, Kuti JL, Nicolau DP. 2005. Antimicrobial management of complicated skin and skin structure infections in the era of emerging resistance. Surg Infect, 6:283-95. 
